\title{
Development of a Model to Predict Healing of Chronic Wounds Within 12 Weeks
}

\begin{abstract}
Sang Kyu Cho, Soeren Mattke, ${ }^{2, *}$ Hanna Gordon, ${ }^{3}$ Mary Sheridan, ${ }^{3}$ and William Ennis ${ }^{3,4}$

${ }^{1}$ Leonard D. Schaeffer Center for Health Policy and Economics, University of Southern California, Los Angeles, California.

${ }^{2}$ Center for Economic and Social Research, University of Southern California, Los Angeles, California.

${ }^{3}$ Healogics, Inc., Jacksonville, Florida.

${ }^{4}$ Department of Surgery, Wound Healing and Tissue Repair Program, University of Illinois Hospital and Health Sciences System, Chicago, Illinois.

Précis: Using both clinically informed and machine learning approaches, we developed a prediction model for chronic wound healing that can form the basis for quality measurement.
\end{abstract}

Objective: Chronic wounds represent a highly prevalent but little recognized condition with substantial implications for patients and payers. While better wound care products and treatment modalities are known to improve healing rates, they are inconsistently used in real-world practice. Predicting healing rates of chronic wounds and comparing to actual rates could be used to detect and reward better quality of care. We developed a prediction model for chronic wound healing. Approach: We analyzed electronic medical records (EMRs) for 620,356 chronic wounds of various etiologies in 261,398 patients from 532 wound care clinics in the United States. Patient-level and wound-level parameters influencing wound healing were identified from prior research and clinician input. Logistic regression and classification tree models to predict the probability of wound healing within 12 weeks were developed using a random sample of $70 \%$ of the wounds and validated in the remaining data.

Results: A total of 365,659 (58.9\%) wounds were healed by week 12. The logistic and classification tree models predicted healing with an area under the curve of 0.712 and 0.717 , respectively. Wound-level characteristics, such as location, area, depth, and etiology, were more powerful predictors than patient demographics and comorbidities.

Innovation: The probability of wound healing can be predicted with reasonable accuracy in real-world data from EMRs.

Conclusion: The resulting severity adjustment model can become the basis for applications like quality measure development, research into clinical practice and performance-based payment.

Keywords: risk scale, prediction, chronic wounds, electronic medical records, real-world data

\section{INTRODUCTION}

Chronic wounds, usually DeFINED as wounds that do not heal within an expected time frame, typi- cally 4-12 weeks, are a growing but little recognized public health challenge. ${ }^{1,2}$ Their prevalence is similar to that of heart failure, affecting 6.5 (c) Sang Kyu Cho, et al., 2020; Published by Mary Ann Liebert, Inc. This Open Access article is distributed under the terms of the Creative Commons License (http://creativecommons.org/licenses/ by/4.0), which permits unrestricted use, distribution, and reproduction in any medium, provided the original work is properly cited. 
million people or $2 \%$ of the population in the United States. ${ }^{3}$ In 2014, estimated Medicare expenditure related to managing chronic wounds and associated complications ranged between $\$ 28.1$ and $\$ 96.8$ billion. ${ }^{4}$ Patients with chronic wounds often suffer loss of productivity, psychological distress, decreased quality of life, and reduced life expectancy. ${ }^{5,6}$

From advanced dressings to growth factors and biologics, substantial progress has been made in product development and treatment approaches for chronic wounds that do not adequately heal with standard treatment. ${ }^{7,8}$ These advanced wound therapies employ various mechanisms to aid wound healing, such as providing a structural scaffold, repairing restorative cellular mechanisms, increasing delivery of oxygen and nutrients, preventing infection, and removing necrotic tissues or exudates. ${ }^{9}$ However, the uptake of these innovations and use of evidence-based treatment practices remain limited. ${ }^{1,2,10}$

For other chronic conditions, payment reform has been proposed as a policy intervention to improve quality of care because the established fee-for-service payment system is increasingly regarded as failing to reward high-quality care. ${ }^{4}$ The Centers for Medicare and Medicaid Services has charted an ambitious course to reorient its payment models to value and has introduced such models for several chronic conditions. ${ }^{11}$ Several so-called Alternative Payment Models, which link reimbursement to overall resource use, patient outcomes, and adherence to clinical practice guidelines, are tested for conditions like heart failure, diabetes, hypertension, and chronic obstructive pulmonary disease. ${ }^{12}$

\section{CLINICAL PROBLEM ADDRESSED}

A critical requirement for the introduction of such value-based payment models is scientifically sound and actionable quality indicators that will trigger rewards or penalties. An obvious choice in chronic wound care is the wound healing rate, as closing wounds is both the intended outcome of treatment and readily observable. But given the heterogeneous etiology of chronic wounds, differences in case mix across wound care centers, and the multitude of factors that can influence healing rates proper severity adjustment is needed to construct a valid quality measure.

Several severity adjustment models have been developed previously, but many suffer from limitations such as small sample size from a single center, applicability to only one wound type, such as diabetic foot ulcer, and low prediction accura- cy. ${ }^{13-18}$ More recent studies leveraged electronic medical records (EMRs) and machine learning techniques to improve prediction accuracy, but their usability as a quality measure is handicapped by data-driven variable selection and incorporation of information on initial healing observed during follow-up visits. ${ }^{19,20}$

The objective of our study is to develop and validate a unified severity adjustment model for a broad range of chronic wounds that can form the basis for a quality measure as it is entirely based on routinely collected data from intake visits at wound care clinics.

\section{MATERIALS AND METHODS \\ Dataset}

The data for our analyses came from the EMRs of a large network of wound care centers in 46 states and cover the timeframe from January 1, 2014 to September 25, 2018. A detailed description of the staffing and skill mix of these centers has been published elsewhere. ${ }^{21}$ In short, these centers are run by a wound care management company and staffed by combination of employed and contracted physicians, supported by specialized nurses and program managers. All clinicians are required to attend a 1-week specialty wound care training course and are provided evidence based algorithmic clinical practice guidelines. The clinicians subsequently receive ongoing education, monthly conference calls, and have access to regional and local medical directors for case management support. The centers are hospital-based and most have access to specialty consultants and advanced treatment modalities, like hyperbaric oxygen. Standardized treatment protocols promote adherence to evidence-based care.

The data contain detailed patient level information such as age, sex, smoking status, body mass index, comorbid conditions, and wound level measurements such as length, width, and depth in addition to categorical descriptors such as wound etiology, location, and appearance. Each wound is assessed at intake and every subsequent visit, and treatment modalities are documented. At the end of each visit, the outcome for each wound is documented as healed or not healed.

Our starting sample consisted of EMRs for 356,649 patients with 914,878 unique wounds. We excluded 202,842 (22\%) wounds that were caused by radiation, acute wounding events such as surgery and trauma, and 71,414 (8\%) wounds in patients, who were only seen in the clinic for initial consultation. We removed 20,266 (2\%) wounds 
with implausible dimensions, such as surface areas $>100 \mathrm{~cm}^{2}$ for arterial ulcer and $150 \mathrm{~cm}^{2}$ for other wound types and wounds from patients with missing age and sex.

The final dataset included 620,356 unique wounds from 261,398 patients from 532 wound care clinics. We only used information that was collected during the initial intake for the prediction model and tracked wound outcomes regardless of therapeutic interventions. We selected a training dataset containing a randomly drawn subset of $70 \%(n=434,249)$ of the wounds to develop the model, leaving a validation set with the remaining $30 \%(n=186,107)$ of the wounds for validation of model parameters (Supplementary Fig. S1).

\section{Definition of outcome}

Our outcome is the status of the wound within the first 12 weeks of treatment initiation, coded dichotomously into healed versus not healed, as commonly used in clinical trials of wound treatment. $^{22,23}$ While patients can remain in treatment for longer than 12 weeks, wound healing in that period was no longer considered for the analysis. Following Ennis et al., we applied a modified intent-to-treat approach in that wounds in patients lost to follow-up during the 12 weeks were considered not healed after excluding wounds that were followed for $<7$ days. $^{21}$

The determination of healing is made by the treating clinician during each visit based on the following criteria: (1) wound has zero wound measurements, that is, it is completely covered with a full layer of epithelium and no longer has exudate; (2) wound has received a flap procedure and presents post procedure with complete take; (3) wound has received a graft procedure and presents post procedure with complete success; and (4) wound margins have been approximated and sutured to facilitate closure and wound has zero measurements.

\section{Selection of predictor variables}

We combined a review of the published literature on prediction models for wound healing and clinical input to select potential predictor variables. We grouped predictor variables into three categories. The first category represented demographic characteristics, such as age, sex, and smoking status, the second patient level clinical characteristics, such as comorbid conditions and the number of wounds, which were identified based on International Classification of Diseases codes (Supplementary Table S1) or clinical notes, and the third, wound level characteristics, such as area, location, and etiology.
Following Shaw et al., wound area was calculated from measured width and length assuming an elliptical shape. ${ }^{24}$ Physical depth of a wound was estimated by the treating clinicians, and the "anatomical" depth (i.e., degree of tissue penetration) was categorized as partial thickness (including dermis and epidermis but not the fascia), full thickness (extension through the fascia into subcutaneous structure), or unknown thickness based on a classification presented in the Supplementary Data. We refer to this variable as "wound classification." Variables that were infrequently coded were not considered for the model. For example, prior studies found ankle brachial index, nutritional status, glycated hemoglobin $\left(\mathrm{HbA}_{1 \mathrm{c}}\right)$, and antibiotic therapy to be predictors of wound healing, but they were infrequently documented in our dataset. ${ }^{25-27}$ While the wound care centers usually collect such information during a patient's intake assessment, it was documented as free text in the EMRs and could not be leveraged for our analyses. ICD-9/10 codes and classification of wound stages are presented in Supplementary Tables S1 and S2.

\section{Model construction}

We used logistic regression models to predict the probability of a wound being healed by the end of week 12 as a function of our three categories of variables: demographic characteristics, patient level clinical characteristics, and wound characteristics. Using the training dataset, we entered variables individually and retained only those that increased the predictive accuracy of the model, as the large sample size implied that many variables were statistically significantly correlated with our outcome without contributing meaningfully to the predictive accuracy.

We added variables in a stepwise fashion and examined their contribution to model performance using area under the curve (AUC) and the Akaike information criterion (AIC). The AUC is a measure of how well the model predicts the outcome. ${ }^{17}$ An AUC of 0.5 means the model performs no better than a random guess, while an AUC of 1.0 means perfect prediction, and values above 0.7 are regarded as acceptable model fit. ${ }^{28}$ Adding variables will always increase predictive power, but also model complexity and risk of "overfitting," that is, optimizing the model to reflect the information in a specific dataset, while making it less generalizable to different datasets. Hence, the AIC was used to assess the contribution of a variable to the informational quality of the model as it increases, if a variable contributes limited explanatory power relative to its contribution to model fit. ${ }^{29}$ 
Having selected variables based on examination of AUC and AIC, we constructed three logistic models of ascending complexity: the first model contained only demographic characteristics, the second model added patient-level clinical characteristics, and the final model wound characteristics.

As the magnitude of predicted odds ratios in logistic regression models cannot tell the extent to which each variable drives the prediction, we used a classification tree model with all variables of the final model to further assess the contribution of each explanatory variable. ${ }^{30}$ The classification tree model is a machine learning approach that recursively splits the data into increasingly homogeneous groups using combinations of predictor variables. For example, the algorithm might determine that all wounds below a certain area in nondiabetic women have healed or that pressure ulcers with full thickness in elderly patients that lasted $>30$ days before admissions did not heal. In other words, the final nodes in the classification tree contain mostly healed or not healed wounds after the data have been split multiple times using combinations of predictor variables. Classification trees can outperform logistic models if there are strong interactions between predictor variables. ${ }^{31}$

The relative variable importance metric derived from such classification tree models is a number between 0 and 1 . It is calculated in two steps. First, importance of each variable is measured based on the change in the sum of the squares of residuals (i.e., difference in predicted and actual values) when a predictor variable is used to split a node. Then, relative importance of each variable is calculated by dividing each variable's importance by the highest variable importance among all predictors. ${ }^{32}$ Like logistic models, our classification tree model was developed and validated using $70 \%$ and $30 \%$ random samples, respectively.

All statistical analyses were conducted using SAS 9.4 (SAS Institute, Cary, NC). Logistic models and classification tree were created using PROC LOGISTIC and PROC HPSPLIT commands, respectively. ${ }^{33}$ The study was reviewed and determined to qualify for the Human Research Protection Program Flexibility Policy by the Institutional Review Board of the University of Southern California (UP-18-00477).

\section{RESULTS}

Table 1 compares characteristics between wounds that healed and wounds that failed to heal by the end of week 12 . About $59 \%$ of wounds healed by the end of week 12. Because of the large
Table 1. Comparison of healed versus not-healed wounds

\begin{tabular}{|c|c|c|c|}
\hline & \multicolumn{3}{|c|}{ Wound Status at 12th Week } \\
\hline & $\begin{array}{c}\text { Healed } \\
(\mathrm{n}=365,659 ; \\
58.9 \%), \mathrm{n}(\%)\end{array}$ & $\begin{array}{l}\text { Not Healed } \\
(\mathrm{n}=254,697 \\
41.1 \%), \mathrm{n}(\%)\end{array}$ & $\mathrm{p}$ \\
\hline \multicolumn{4}{|l|}{ Demographic characteristics } \\
\hline Age category & & & $<0.0001$ \\
\hline$<55$ & $84,840(23.2)$ & $61,769(24.3)$ & \\
\hline $55-64$ & $82,030(22.4)$ & $57,910(22.7)$ & \\
\hline $65-74$ & $89,277(24.4)$ & $59,054(23.2)$ & \\
\hline$>75$ & $109,512(30.0)$ & $75,964(29.8)$ & \\
\hline BMI category & & & $<0.0001$ \\
\hline$<18.5$ & $4,180(1.1)$ & $4,717(1.9)$ & \\
\hline $18.5-24$ & 30,725 (8.4) & $26,925(10.6)$ & \\
\hline $25-29$ & $38,736(10.6)$ & $28,764(11.3)$ & \\
\hline$>30$ & $99,017(27.1)$ & $54,349(21.3)$ & \\
\hline Missing & $193,001(52.8)$ & $139,942(54.9)$ & \\
\hline Sex (female) & $153,052(41.9)$ & $108,145(42.5)$ & $<0.0001$ \\
\hline Palliative & $5,176(1.4)$ & $10,754(4.2)$ & $<0.0001$ \\
\hline Smoking status & & & $<0.0001$ \\
\hline Never smoker & $118,640(32.5)$ & $77,640(30.5)$ & \\
\hline Former smoker & $93,523(25.6)$ & $63,343(24.9)$ & \\
\hline Current smoker & $41,226(11.3)$ & $33,527(13.2)$ & \\
\hline Unknown & $112,270(30.7)$ & $80,187(31.5)$ & \\
\hline \multicolumn{4}{|l|}{ Clinical characteristics } \\
\hline Alzheimer's disease/dementia & $13,656(3.7)$ & $13,920(5.5)$ & $<0.0001$ \\
\hline Coronary artery disease & $61,053(16.7)$ & $46,068(18.1)$ & $<0.0001$ \\
\hline Congestive heart failure & $55,559(15.2)$ & $40,749(16.0)$ & $<0.0001$ \\
\hline $\begin{array}{l}\text { Chronic obstructive pulmonary } \\
\text { disease }\end{array}$ & $43,703(12.0)$ & $30,403(11.9)$ & 0.8586 \\
\hline Diabetes & $220,893(60.4)$ & $154,372(60.6)$ & 0.1120 \\
\hline Peripheral vascular diseases & $77,959(21.3)$ & $67,471(26.5)$ & $<0.0001$ \\
\hline Quadri/paraplegia & $7,681(2.1)$ & $11,872(4.7)$ & $<0.0001$ \\
\hline Hypertension & $189,764(51.9)$ & $128,880(50.6)$ & $<0.0001$ \\
\hline Number of concurrent wounds & & & $<0.0001$ \\
\hline 1 & $169,589(46.4)$ & $91,799(36.0)$ & \\
\hline 2 & 70,977 (19.4) & $52,024(20.4)$ & \\
\hline 3 or more & $125,093(34.2)$ & $110,874(43.5)$ & \\
\hline \multicolumn{4}{|l|}{ Wound characteristics } \\
\hline Wound area $\left(\mathrm{cm}^{2}\right)($ mean/SD) & $6.4(16.5)$ & $9.7(19.0)$ & $<0.0001$ \\
\hline Wound depth (mm) (mean/SD) & $2.1(5.0)$ & $4.1(8.1)$ & $<0.0001$ \\
\hline Wound location & & & $<0.0001$ \\
\hline Pelvic & $31,801(8.7)$ & $32,759(12.9)$ & \\
\hline Upper leg & $13,345(3.7)$ & $6,980(2.7)$ & \\
\hline Lower leg & $153,995(42.1)$ & $70,559(27.7)$ & \\
\hline Foot & $81,564(22.3)$ & $87,863(34.5)$ & \\
\hline Toe & $53,544(14.6)$ & $39,462(15.5)$ & \\
\hline Amputation site & $3,614(1.0)$ & 4,199 (1.7) & \\
\hline Other & $27,799(7.6)$ & $12,875(5.1)$ & \\
\hline Wound classification & & & $<0.0001$ \\
\hline Full thickness & $157,903(43.2)$ & $132,153(51.9)$ & \\
\hline Partial thickness & $121,364(33.2)$ & $47,575(18.7)$ & \\
\hline Superficial & $30,290(8.3)$ & $16,710(6.6)$ & \\
\hline Unknown & $56,102(15.3)$ & $58,259(22.9)$ & \\
\hline Wound etiology & & & $<0.0001$ \\
\hline Arterial ulcer & $8,844(2.4)$ & $14,842(5.8)$ & \\
\hline Diabetic ulcer & $127,129(34.8)$ & $98,792(38.8)$ & \\
\hline Pressure ulcer & $54,500(14.9)$ & $57,514(22.6)$ & \\
\hline Venous ulcer & $97,047(26.5)$ & $44,035(17.3)$ & \\
\hline Other & $78,139(21.4)$ & $39,514(15.5)$ & \\
\hline Necrotic wound tissue & 97,392 (26.6) & $88,797(34.9)$ & $<0.0001$ \\
\hline Infected wound & $124,198(34.0)$ & $99,026(38.9)$ & $<0.0001$ \\
\hline Heavily exuding wound & $98,921(27.1)$ & $81,616(32.0)$ & $<0.0001$ \\
\hline Eschar formation & $24,500(6.7)$ & $28,960(11.4)$ & $<0.0001$ \\
\hline
\end{tabular}

BMI, body mass index; SD, standard deviation. 
Table 2. Comparison of model performance for different specifications

\begin{tabular}{lcc}
\hline Model Type & AUC & AIC \\
\hline Model 1: Demographics only & 0.556 & $24,9547.7$ \\
Model 2: Demographics + clinical characteristics & 0.605 & $24,5548.4$ \\
Model 3: Demographics + clinical + wound characteristics & 0.712 & $22,5519.3$
\end{tabular}

AIC, Akaike information criterion; AUC, area under the curve.

sample size, all variables, except for patient's history of diabetes and chronic obstructive pulmonary disease, showed statistically significant differences, even when the absolute magnitude of the differences was small. Healed wounds were smaller in area and depth and associated with lower prevalence of comorbidities such as Alzheimer's disease or dementia, coronary artery disease, and peripheral vascular diseases. Pressure ulcers and arterial ulcers were less likely to heal than wounds of other etiology.

The comparison of AUC and AIC for the three logistic regression models is shown in Table 2. The predictive accuracy of a model using only demographic variables was limited with an AUC of 0.556 and adding indicators for comorbid conditions only improved the AUC to 0.605 . The addition of variables capturing wound characteristics resulted in substantial improvement in predictive accuracy with an AUC of 0.712. The AIC decreased with the addition of comorbidity and wound characteristic variables, indicating that the additional sets of variables were informative in predicting wound healing. AUCs and AICs of three models are presented in Fig. 1. The comparison of AUCs and AICs between training and validation sets is presented in Supplementary Table S3.

Table 3 shows the estimated odds ratios for the final model that used all wound and patient level characteristics. Pressure ulcers, arterial ulcers, full-thickness wounds, and wounds in patients with multiple concurrent wounds were less likely to heal by the end of week 12 .

The analysis of the relative variable importance based on the classification tree model shows a similar pattern. As shown in Table 4, wound location had the highest variable importance in predicting wound healing, and the importance of the other variables is presented relative to it. Seven of the ten most important predictors were wound level characteristics and eight of the ten least important predictors were patient-level characteristics. Patient level characteristics had a relative variable importance of 0.2 or less, whereas wound characteristics had a relative variable importance around or above 0.5 . The classification tree model achieved an AUC of 0.717.

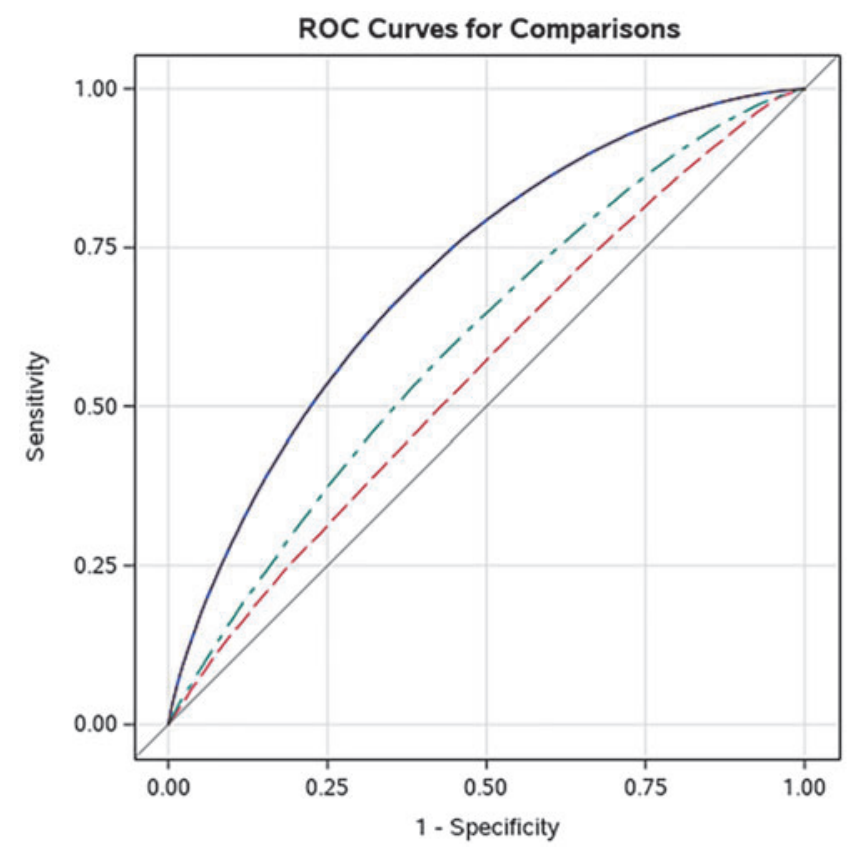

Figure 1. Comparison of area under the receiver operator curves for different model specifications. Purple: Model based on demographics + clinical characteristics + wound characteristics. Green: Model based on demographics + clinical characteristics. Red: Model based on demographics only. ROC, receiver operating characteristic.

\section{DISCUSSION}

Our study used data from a large EMR to predict the probability of wound healing as a function of patient and wound characteristics with both clinically informed and machine learning approaches. Both methods achieved acceptable predictive accuracy with AUCs above 0.70. Our results are consistent with previous findings that wound level characteristics are better predictors of wound healing than patient level characteristics, such as demographic information and comorbidities. ${ }^{12-14,30}$

Our model's performance is comparable or higher than previously published models. For example, using the U.S. Wound Registry that combines EMRs from over 50 wound care clinics, Horn et al. ${ }^{34}$ reported AUCs between 0.594 and 0.708 and Fife $e t a l .{ }^{35}$ reported an AUC of 0.648. Using EMRs from a large health system, Margolis et al. achieved AUCs of 0.70 and $0.71 .^{36} \mathrm{An}$ important distinction, however, is that our model applies to a broad range of wound etiologies, whereas other models were restricted to a single etiology such as diabetic neuropathic foot ulcers or venous leg ulcers.

Another important distinction is that our model exclusively relies on data collected at the patient's initial intake exam, whereas other studies added information on wound healing progress from sub- 
Table 3. Estimated odds ratios from full logistic regression model (Model 3)

\begin{tabular}{|c|c|c|c|c|}
\hline \multirow{3}{*}{$\begin{array}{l}\text { Variable } \\
\text { (Comparator vs. Reference) } \\
\text { Depth }\end{array}$} & \multicolumn{4}{|c|}{ Odds Ratio } \\
\hline & \multirow{2}{*}{$\begin{array}{c}\begin{array}{c}\text { Point } \\
\text { Estimate }\end{array} \\
0.943\end{array}$} & \multicolumn{2}{|c|}{$\begin{array}{l}\text { 95\% Confidence } \\
\text { Interval }\end{array}$} & \multirow{2}{*}{$\frac{p}{<0.0001}$} \\
\hline & & 0.942 & 0.945 & \\
\hline Wound surface area & 0.987 & 0.986 & 0.987 & $<0.0001$ \\
\hline \multicolumn{5}{|l|}{ Age category, years } \\
\hline$<55$ & \multicolumn{4}{|c|}{ (Reference) } \\
\hline $55-64$ & 0.999 & 0.980 & 1.019 & 0.0110 \\
\hline $65-74$ & 1.028 & 1.008 & 1.049 & 0.0245 \\
\hline$>75$ & 1.031 & 1.011 & 1.052 & 0.0064 \\
\hline \multicolumn{5}{|l|}{ BMI category } \\
\hline $18.5-24$ & \multicolumn{4}{|c|}{ (Reference) } \\
\hline $25-29$ & 1.091 & 1.060 & 1.123 & $<0.0001$ \\
\hline$>30$ & 1.277 & 1.244 & 1.311 & $<0.0001$ \\
\hline$<18.5$ & 0.829 & 0.782 & 0.878 & $<0.0001$ \\
\hline Missing & 0.968 & 0.945 & 0.992 & $<0.0001$ \\
\hline Palliative (yes vs. no) & 0.414 & 0.397 & 0.433 & $<0.0001$ \\
\hline Sex (male vs. female) & 1.125 & 1.110 & 1.140 & $<0.0001$ \\
\hline \multicolumn{5}{|l|}{ Smoking status } \\
\hline Never smoker & \multicolumn{4}{|c|}{ (Reference) } \\
\hline Current smoker & 0.868 & 0.849 & 0.889 & $<0.0001$ \\
\hline Former smoker & 0.987 & 0.969 & 1.005 & $<0.0001$ \\
\hline Unknown & 0.817 & 0.800 & 0.834 & $<0.0001$ \\
\hline Dementia/Alzheimer's (yes vs. no) & 0.820 & 0.794 & 0.848 & $<0.0001$ \\
\hline Coronary artery disease (yes vs. no) & 0.981 & 0.962 & 1.000 & 0.0466 \\
\hline Congestive heart failure (yes vs. no) & 0.903 & 0.885 & 0.921 & $<0.0001$ \\
\hline $\begin{array}{l}\text { Chronic obstructive pulmonary } \\
\text { disease (yes vs. no) }\end{array}$ & 1.029 & 1.006 & 1.051 & 0.0115 \\
\hline Diabetes (yes vs. no) & 1.114 & 1.095 & 1.134 & $<0.0001$ \\
\hline $\begin{array}{l}\text { Peripheral vascular } \\
\text { diseases (yes vs. no) }\end{array}$ & 0.774 & 0.761 & 0.787 & $<0.0001$ \\
\hline Quadri/paraplegia (yes vs. no) & 0.661 & 0.634 & 0.688 & $<0.0001$ \\
\hline Hypertension (yes vs. no) & 1.048 & 1.031 & 1.066 & $<0.0001$ \\
\hline \multicolumn{5}{|l|}{ Number of concurrent wounds } \\
\hline 1 & \multicolumn{4}{|c|}{ (Reference) } \\
\hline 2 & 0.728 & 0.715 & 0.741 & $<0.0001$ \\
\hline$>2$ & 0.567 & 0.559 & 0.576 & $<0.0001$ \\
\hline \multicolumn{5}{|l|}{ Wound location } \\
\hline Foot & \multicolumn{4}{|c|}{ (Reference) } \\
\hline Pelvic & 1.388 & 1.349 & 1.429 & $<0.0001$ \\
\hline Upper leg & 2.063 & 1.982 & 2.147 & $<0.0001$ \\
\hline Lower leg & 1.966 & 1.928 & 2.005 & $<0.0001$ \\
\hline Toe & 1.319 & 1.292 & 1.346 & $<0.0001$ \\
\hline Amputation site & 1.117 & 1.054 & 1.183 & $<0.0001$ \\
\hline Other & 2.019 & 1.953 & 2.086 & $<0.0001$ \\
\hline \multicolumn{5}{|l|}{ Wound classification } \\
\hline Superficial & \multicolumn{4}{|c|}{ (Reference) } \\
\hline Full thickness & 0.501 & 0.485 & 0.517 & $<0.0001$ \\
\hline Partial thickness & 0.886 & 0.856 & 0.918 & $<0.0001$ \\
\hline Unknown & 0.388 & 0.376 & 0.402 & $<0.0001$ \\
\hline \multicolumn{5}{|l|}{ Wound type } \\
\hline Diabetic ulcer & & (Refe & nce) & \\
\hline Arterial ulcer & 0.669 & 0.644 & 0.694 & $<0.0001$ \\
\hline Other & 1.609 & 1.569 & 1.650 & $<0.0001$ \\
\hline Pressure ulcer & 0.857 & 0.832 & 0.883 & $<0.0001$ \\
\hline Venous ulcer & 1.460 & 1.425 & 1.495 & $<0.0001$ \\
\hline Necrotic wound tissue (yes vs. no) & 0.784 & 0.771 & 0.797 & $<0.0001$ \\
\hline Infected wound (yes vs. no) & 0.887 & 0.874 & 0.901 & $<0.0001$ \\
\hline Heavily exuding wound (yes vs. no) & 0.909 & 0.894 & 0.925 & $<0.0001$ \\
\hline Eschar formation (yes vs. no) & 0.790 & 0.770 & 0.811 & $<0.0001$ \\
\hline
\end{tabular}

Table 4. Relative importance of variables

\begin{tabular}{lc}
\hline & Relative Importance \\
\hline Would location & 1.0000 \\
Wound surface area & 0.9260 \\
Wound classification & 0.7329 \\
Wound etiology & 0.5550 \\
Number of concurrent wounds & 0.4919 \\
Depth & 0.3427 \\
Palliative & 0.2441 \\
Necrotic wound tissue & 0.2030 \\
Peripheral vascular disease & 0.2009 \\
BMl & 0.1846 \\
Age & 0.1198 \\
Eschar formation & 0.1145 \\
Sex & 0.1128 \\
Smoking status & 0.0943 \\
Infected wound & 0.0799 \\
Diabetes & 0.0585 \\
Heavily exuding wound & 0.0424 \\
Ouadri/paraplegia & 0.0401 \\
Alzheimer's disease/dementia & 0.0343 \\
Congestive heart failure & 0.0342 \\
Coronary artery disease & 0.0311 \\
Chronic obstructive pulmonary disease & 0.0296 \\
Hypertension & 0.0262 \\
\hline
\end{tabular}

AUC: 0.717 .

sequent visits to increase predictive accuracy. Jung et $a l$., for example, used a machine learning approach and constructed a predictive model with an AUC of 0.842 , but the change in wound dimensions between the first and second visit contributed substantially to the model's performance. ${ }^{19}$ Similarly, Horn et al. demonstrated that adding information from subsequent visits increased the AUCs to between 0.615 and 0.726 compared to their abovementioned models with AUCs between 0.594 and 0.708 that only used data collected during patient intake. ${ }^{34}$ It is to be expected that the initial healing is a strong predictor of future healing and may help to identify patients, who show insufficient progress in wound healing and may require additional interventions. Thus, models including information on healing progress have utility for clinical decision making. However, the objective of our work was also to use healing rates to compare quality of care between centers. As healing progress will be influenced by initial treatment, we would argue that including it in a model might result in confounding. ${ }^{37}$

Although an AUC $>0.70$ represents acceptable predictive accuracy of our model, the results point to room for improvement. A logical focus would be information on wound characteristics, as our results show that they are better predictors of healing than patient characteristics. One could explore additional wound level characteristics, such as presence of biofilm, bioburden or activity patterns 
of metalloproteases, as those have been shown to impact wound healing. ${ }^{38,39}$

In addition, as our results indicate that wound dimensions are an influential predictor of healing, increasing the precision with which they are measured and thus reducing random measurement error might improve predictive accuracy. At the moment, the wound care centers contributing to our data typically estimate surface area based on maximum width and length and approximate depth. Especially for larger and irregularly shaped wounds, this approach can lead to error compared to methods like three-dimensional digital imaging techniques. ${ }^{40}$

Furthermore, more complete documentation of patient-level characteristics could improve predictive accuracy. Body mass index and smoking status, for example, are known predictors of wound healing but were missing in $54 \%$ and $31 \%$ in our data, respectively. ${ }^{41}$ Similarly, measures of metabolic control, like $\mathrm{HbA}_{1 \mathrm{c}}$, ankle brachial index for perfusion or skin temperature for inflammation are correlated with wound healing but not yet documented routinely in our data. ${ }^{25,36,42}$

Conversely, we have no evidence that increasing the complexity of the model structure will raise predictive accuracy. We tested several interaction effects with only marginal improvement in the AUC. For example, adding interaction terms for wound area $\times$ depth and wound location $\times$ classification to the final model improved the AUC by only 0.001. Thus, we have no evidence that the interrelationships between predictive variables improve model performance. This result is consistent with the observation that the classification tree model, whose predictive power relies heavily on interactions between predictor variables, had a similar AUC as the final logistic model. Similarly, the use of multi-level models that accounted for clustering of wounds by patients and patients by centers did not improve predictive power meaningfully. In addition, our results suggest that a more parsimonious prediction model using solely wound level characteristics performs comparably to our current model. We tested a logistic model with wound characteristics only, and the model achieved an AUC of 0.689 .

Our analysis has important limitations. First, while our dataset is large, it only contains data from one wound care EMR system and would have to be validated, ideally prospectively, with data from other settings. Second, our data have been collected under real-world conditions without validation and/or formal assessment of coding quality and may be subject to measurement error. However, we would argue that this error is likely to be random and thus reduce rather than overstate our predictive accuracy. Lastly, some of our design decisions, such as exclusion of wounds with implausible dimensions and creation of a unified grading scheme, may have introduced error or even bias. As with all predictive models, performance needs to be tracked over time to account for changes in coding practice and clinical care.

\section{INNOVATION}

Our findings show that wound healing can be predicted based on real-world data from EMRs and a model based on prior evidence and clinical reasoning. Our model can inform clinical decision making and form the basis for applications like quality measure development, future research into clinical practice to determine sources of variability, and performance-based payment.

\section{ACKNOWLEDGMENTS AND FUNDING SOURCES}

This study was conducted under a contract from Healogics, Inc.

\section{AUTHOR DISCLOSURE AND GHOSTWRITING}

S.M. serves on the board of directors of Senscio Systems, Inc., and the scientific advisory board of AiCure Technologies, Boston Millennia Partners, and Zano Zano Healthcare Services. He has received consulting fees from AARP, Biotronik, Bristol-Myers Squibb, Eisai, and Defined Health. H.G., M.S., and W.E. are employees of Healogics, Inc. S.K.C. reports no disclosures.

\section{ABOUT THE AUTHORS}

Sang Kyu Cho, PharmD, MPH, is a $\mathrm{PhD}$ candidate in health economics at Schaeffer Center 
for Health Policy and Economics at University of Southern California. Soeren Mattke, MD, MSc, is a director at Center for Center for Economic and Social Research at University of Southern California. Hanna Gordon, PhD, was an employee at Healogics, Inc. at the time this study was conducted. Mary Sheridan, PhD, is an employee of Healogics, Inc. William Ennis, DO, MBA, MMM, is the section chief of Wound Healing and Tissue Repair Program at University of Illinois.

\section{FUNDING INFORMATION}

Supported by a contract from Healogics, Inc. to the University of Southern California.

\section{SUPPLEMENTARY MATERIAL}

Supplementary Table S1

Supplementary Table S2

Supplementary Table S3

Supplementary Figure S1

\section{REFERENCES}

1. Lazarus GS, Cooper DM, Knighton DR, et al. Definitions and guidelines for assessment of wounds and evaluation of healing. Wound Repair Regen 1994;2:165-170.

2. Coleman $S$, Nelson EA, Vowden P, et al. Development of a generic wound care assessment minimum data set. J Tissue Viability 2017;26: 226-240.

3. Fife CE, Carter MJ, Walker D, Thomson B. Wound care outcomes and associated cost among patients treated in US out-patient wound centers: data from the US wound registry. Wounds 2012; 24:10-17

4. Nussbaum SR, Carter MJ, Fife CE, et al. An economic evaluation of the impact, cost, and medicare policy implications of chronic nonhealing wounds. Value Health 2018;21:27-32.

5. Snyder RJ, Fife C, Moore Z. Components and quality measures of DIME (devitalized tissue, infection/inflammation, moisture balance, and edge preparation) in wound care. Adv Skin Wound Care 2016;29:205-215.

6. Fortington LV, Geertzen JH, van Netten JJ, Postema K, Rommers GM, Dijkstra PU. Short and long term mortality rates after a lower limb amputation. Eur J Vasc Endovasc Surg 2013;46: 124-131.

7. Murphy PS, Evans GR. Advances in wound healing: a review of current wound healing products. Plast Surg Int 2012;2012:190436

8. Gainza G, Villullas S, Pedraz JL, Hernandez RM, Igartua M. Advances in drug delivery systems (DDSs) to release growth factors for wound healing and skin regeneration. Nanomedicine 2015;11: 1551-1573.

9. Greer N, Foman NA, MacDonald R, et al. Advanced wound care therapies for nonhealing diabetic, venous, and arterial ulcers: a systematic review. Ann Intern Med 2013;159:532-542.

10. Warriner RA, 3rd, Carter MJ. The current state of evidence-based protocols in wound care. Plast Reconstr Surg 2011;127(Suppl. 1):144S$153 \mathrm{~S}$
11. Hospital Readmissions Reduction Program (HRRP). Centers for Medicare and Medicaid Services. https://www.cms.gov/medicare/medicare-fee-forservice-payment/acuteinpatientpps/readmissionsreduction-program.html (last accessed March 15, 2019).

12. Innovation Models. Centers for Medicare and Medicaid Services. https://innovation.cms.gov/ initiatives/\#views=models (last accessed March 15, 2019).

13. Ince P, Game FL, Jeffcoate WJ. Rate of healing of neuropathic ulcers of the foot in diabetes and its relationship to ulcer duration and ulcer area. Diabetes Care 2007;30:660-663.

14. Margolis DJ, Allen-Taylor L, Hoffstad O, Berlin JA. Diabetic neuropathic foot ulcers and amputation. Wound Repair Regen 2005;13:230-236.

15. Margolis DJ, Allen-Taylor L, Hoffstad O, Berlin JA. Diabetic neuropathic foot ulcers: the association of wound size, wound duration, and wound grade on healing. Diabetes Care 2002;25:1835-1839.

16. Margolis DJ, Allen-Taylor L, Hoffstad O, Berlin JA. Diabetic neuropathic foot ulcers: predicting which ones will not heal. Am J Med 2003;115:627-631.

17. Kurd SK, Hoffstad OJ, Bilker WB, Margolis DJ. Evaluation of the use of prognostic information for the care of individuals with venous leg ulcers or diabetic neuropathic foot ulcers. Wound Repair Regen 2009;17:318-325.

18. Guihan M, Sohn MW, Bauman WA, et al. Difficulty in identifying factors responsible for pressure ulcer healing in veterans with spinal cord injury. Arch Phys Med Rehabil 2016;97:2085.e1-2094.e1.

19. Jung K, Covington S, Sen CK, et al. Rapid identification of slow healing wounds. Wound Repair Regen 2016;24:181-188.

20. Jung K, Shah NH. Implications of non-stationarity on predictive modeling using EHRs. J Biomed Inform 2015;58:168-174.

21. Ennis WJ, Hoffman RA, Gurtner GC, Kirsner RS, Gordon HM. Wound healing outcomes: using big data and a modified intent-to-treat method as a metric for reporting healing rates. Wound Repair Regen 2017;25:665-672.

22. Arundel C, Buckley $\mathrm{H}$, Clarke E, et al. Negative pressure wound therapy versus usual care for Surgical Wounds Healing by Secondary Intention (SWHSI trial): study protocol for a randomised controlled pilot trial. Trials 2016;17:535.

23. Marston WA, Hanft J, Norwood P, Pollak R. The efficacy and safety of dermagraft in improving the healing of chronic diabetic foot ulcers: results of a prospective randomized trial. Diabetes Care 2003; 26:1701-1705.

24. Shaw J, Hughes CM, Lagan KM, Bell PM, Stevenson MR. An evaluation of three wound measurement techniques in diabetic foot wounds. Diabetes Care 2007;30:2641-2642.

25. Brownrigg JR, Hinchliffe RJ, Apelqvist J, et al. Performance of prognostic markers in the prediction of wound healing or amputation among patients with foot ulcers in diabetes: a systematic review. Diabetes Metab Res Rev 2016;32/Suppl. 1):128-135.

26. Zimny S, Pfohl M. Healing times and prediction of wound healing in neuropathic diabetic foot ulcers: a prospective study. Exp Clin Endocrinol Diabetes 2005;113:90-93

27. Jones KR, Fennie K, Lenihan A. Chronic wounds: factors influencing healing within 3 months and nonhealing after 5-6 months of care. Wounds 2007;19:51-63.

28. Zou KH, O'Malley AJ, Mauri L. Receiver-operating characteristic analysis for evaluating diagnostic tests and predictive models. Circulation 2007;115: 654-657.

29. Arnold TW. Uninformative parameters and model selection using Akaike's information criterion. J Wildl Manag 2010;74:1175-1178.

30. Tonidandel S, LeBreton JM. Relative importance analysis: a useful supplement to regression analysis. J Bus Psychol 2011;26:1-9.

31. Culter DR. What statisticians should know about machine learning. Paper 883-2017. SAS Global 
Forum 2017. https://support.sas.com/resources/ papers/proceedings17/0883-2017.pdf (last accessed March 15, 2019).

32. SAS/STAT ${ }^{\circledR} 14.1$ User's guide the HPSPLIT procedure. SAS Institute. https://support.sas.com/ documentation/onlinedoc/stat/141/hpsplit.pdf (last accessed March 15, 2019).

33. Rothman L. Predictive Modeling with SAS (for Health). SAS Institute. https://www.sas.com/content/dam/ SAS/en_ca/User\%20Group\%20Presentations/ Victoria-User-Group/Rothman-PredictiveModeling forHealth.pdf (last accessed March 15, 2019).

34. Horn SD, Fife CE, Smout RJ, Barrett RS, Thomson B. Development of a wound healing index for patients with chronic wounds. Wound Repair Regen 2013;21:823-832.

35. Fife CE, Horn SD, Smout RJ, Barrett RS, Thomson B. A predictive model for diabetic foot ulcer outcome: the wound healing index. Adv Wound Care (New Rochelle) 2016;5:279-287.
36. Margolis DJ, Allen-Taylor L, Hoffstad O, Berlin JA. The accuracy of venous leg ulcer prognostic models in a wound care system. Wound Repair Regen 2004;12:163-168.

37. Sheehan P, Jones P, Caselli A, Giurini JM, Veves A. Percent change in wound area of diabetic foot ulcers over a 4-week period is a robust predictor of complete healing in a 12-week prospective trial. Diabetes Care 2003;26:1879-1882.

38. Tuttle MS. Association between microbial bioburden and healing outcomes in venous leg ulcers: a review of the evidence. Adv Wound Care (New Rochelle) 2015;4:1-11.

39. McCarty SM, Percival SL. Proteases and delayed wound healing. Adv Wound Care (New Rochelle) 2013:2:438-447.

40. Bilgin $M$, Gunes UY. A comparison of 3 wound measurement techniques: effects of pressure ulcer size and shape. J Wound Ostomy Continence Nurs 2013:40:590-593.
41. Anderson K, Hamm RL. Factors that impair wound healing. J Am Coll Clin Wound Spec 2012 4:84-91.

42. Lavery LA, Petersen BJ, Linders DR, Bloom JD, Rothenberg GM, Armstrong DG. Unilateral remote temperature monitoring to predict future ulceration for the diabetic foot in remission. BMJ Open Diabetes Res Care 2019;7:e000696.

\section{Abbreviations and Acronyms}

$A U C=$ area under the curve

$A I C=$ Akaike information criterion

$\mathrm{BMI}=$ body mass index

EMRs = electronic medical records

$\mathrm{HbA}_{1 \mathrm{c}}=$ glycated hemoglobin

$\mathrm{ROC}=$ receiver operating characteristic

$\mathrm{SD}=$ standard deviation 